\title{
Sirt1/Nrf2 signalling pathway prevents cognitive impairment in diabetic rats through anti-oxidative stress induced by miRNA-23b-3p expression
}

\author{
JINHUA HAN $^{1}$, XIAONING LIU ${ }^{2}$, YINGZHI LI ${ }^{2}$, JUN ZHANG $^{2}$ and HAICHI YU ${ }^{2}$ \\ Departments of ${ }^{1}$ Radiotherapy and ${ }^{2}$ Orthopaedics, The Second Hospital of \\ Jilin University, Changchun, Jilin 130021, P.R. China
}

Received July 23, 2017; Accepted December 12, 2017

DOI: $10.3892 / \mathrm{mmr} .2018 .8876$

\begin{abstract}
In the present study the exact roles and mechanisms underlying the effect of miRNA-23b-3p on the cognitive impairment of diabetic rats were investigated. The in vivo model of diabetes was established in Wistar rats via a single injection of streptozotocin (STZ). Cognitive function was evaluated using a Morris water maze test. Oxidative stress was measured using ELISA kits, and the protein expression levels of B-cell lymphoma 2-associated X protein, silent information regulator 1 (SIRT1), nuclear factor erythroid 2-related factor 2 (Nrf2) and GAPDH were measured by western blot analysis. Micro (mi)RNA-23b-3p mimics were employed to increase miRNA-23b-3p expression in the in vitro model. Overexpression of miRNA-23b-3p increased oxidative stress (as indicated by the levels of glutathione peroxidase, glutathione, superoxide dismutase and malondialdehyde) and apoptosis in neurocytes following high-glucose treatment. The overexpression of miRNA-23b-3p also suppressed SIRT1 and Nrf2 expression in neurocytes following high-glucose treatment; it also promoted the SIRT1-induced inhibition of apoptosis and oxidative stress. The promotion of SIRT1 also decreased the effect of miRNA-23b-3p on cognitive impairment in diabetic rats. In conclusion, miRNA-23b-3p prevents the cognitive impairment of diabetic rats via anti-oxidative stress effects and the Sirt1/Nrf2 signaling pathway.
\end{abstract}

\section{Introduction}

The rapid development of human society and economy has rendered great changes in people's ways of work and life (1).

Correspondence to: Mr. Haichi Yu, Department of Orthopaedics, The Second Hospital of Jilin University, 218 Ziqiang Street, Changchun, Jilin 130021, P.R. China

E-mail: yuq279650f@163.com

Key words: microRNA-23b-3p, cognitive impairment, diabetic, silent information regulator 1, nuclear factor erythroid 2-related factor 2
Factors such as aging of social population, obesity, unhealthy living habits and heredity, have given rise to the gradually increased morbidity of diabetes mellitus (DM) (2). DM has become one of the global public health problems severely threatening human health after cardiovascular disease and tumor (2). According to the International Diabetes Federal (IDF) statistics, the world has witnessed approximately 0.246 billion DM patients in 2007 (1). Such fig. will increase to 0.380 billion by 2025 if no comprehensive control measure is carried out. DM epidemiological investigation in China in 1980 and 1994 suggested that DM morbidity in adult was 0.9 and $2.5 \%$, respectively (3).

DM is associated with long course of disease, many complications and high medical expenses, which has greatly affected the patients and the society (4). At present, non-infectious diseases dominated by DM, tumor and cardiovascular disease have become the major causes of global death (5). Therefore, carrying out research on the prevention and treatment of DM and its complications is of great social significance (5). Disorder of sugar, lipid and protein metabolism will induce systemic pathophysiological damage in multiple systems, tissues and organs (5). Moreover, it will also lead to complications in organs like eye, kidney, heart and brain (5). Of them, nervous system complications are the most common, such as DM neuropathy (5). At present, numerous reports regarding DM peripheral neuropathy are available from domestic and foreign medical scientific research (6). Particularly, DM cognitive impairment induced by DM central nervous system injury has been extensively studied.

Silent information regulator 1 (SIRT1) is a nicotinamide adenine dinucleotide (NAD+)-dependent histone deacetylase (7). It can act on histone and multiple non-histones, thus being involved in multiple physiopathological processes like anti-inflammation, anti-oxidative stress, anti-apoptosis and anti-cell aging (8).

miRNA is a big family of small molecule non-coding single-strand RNA with the length of approximately 20-25 basic groups (9). It is formed by a segment of single-strand RNA precursor with hairpin loop structure, which is 70-90 basic groups in length, after Dicer digestion (9). It can regulate gene expression at transcription level or post-transcription level (10). miRNA can bind with the 3'untranslated region 
(UTR) of target message RNA (mRNA), thus exerting its function (10). In addition, it can result in transcription inhibition, and mRNA instability or degradation, and thus affect the pathophysiological processes of diseases (11).

Plenty of miRNAs have been discovered in brain tissue of mammals at present (10). They are related to brain tissue development, neuronal differentiation and senior neurological functions (such as learning and memory) (6). Moreover, they are also associated with diseases like neurodegenerative disease, mental disorder and brain tumor (6). As is found in recent research, miRNAs are also involved in regulating neuron cell cycle control and apoptosis in Alzheimer's disease (AD) and Parkinson's disease (PD) (12). Besides, they also participate in regulating the pathological process of post-ischemic brain injury (12). In this report, we investigated the exact roles and mechanisms of miRNA-23b-3p on cognitive impairment of diabetic rats.

\section{Materials and methods}

Animals. Firstly, male 8-week-old Wistar rats (180-220 g) were purchased from the Laboratory Animal Center of Jilin University, and housed in standard laboratory cages at $22-24^{\circ} \mathrm{C}$ with a 12-h light/dark cycle and were provided free access to food and water. The experimental animals were randomly divided into two groups, the control group $(n=6)$ and the DM group $(n=6)$. DM model group, Wistar rats were induced via a single injection of $50 \mathrm{mg} / \mathrm{kg}$ streptozotocin (STZ; Sigma-Aldrich, Merck KGaA, Darmstadt, Germany) into the left lower abdominal cavity. On 8 week after STZ injection, the rats were anaesthetised with sodium pentobarbital $(35 \mathrm{mg} / \mathrm{kg}$, intraperitoneally; Beijing Propbs Biotechnology, Co., Ltd., Beijing, China). All experimental protocols and procedures were approved by the Animal Care and Ethics Committee of The Second Hospital of Jilin University (Jilin, China).

Next, 18 rats were randomly divided into three groups, the control group $(n=6)$, the DM group $(n=6)$ and SIRT1 group $(n=6)$. SIRT1 group, after injection of STZ for 4 weeks, DM rat was gavaged with $50 \mathrm{mg} / \mathrm{kg}$ of SRT1720 (SIRT1 agonist, MedChemExpress) for 4 weeks.

Haematoxylin and eosin $(H \& E)$ staining. After treatment with SRT1720, rats was anaesthetised with $35 \mathrm{mg} / \mathrm{kg}$ of sodium pentobarbital and sacrificed by decollation (13). Then, brain tissue samples were separated and hippocampus was stripped as reference (14). Hippocampus was fixed by $4 \%$ paraformaldehyde for $24 \mathrm{~h}$, and sample was cleared with xylene and routinely embedded in paraffin. Tissue was cut into Serial coronal sections $(10 \mu \mathrm{m})$, and then deparaffinised, rehydrated and then stained with H\&E at room temperature for $15 \mathrm{~min}$. Samples were observed via light microscopy (Olympus, Tokyo, Japan).

RNA extraction for microRNA expression analysis. Total RNA was extracted from PC12 cells or hippocampus tissue using miRNeasy ${ }^{\circledR}$ Mini kit (Qiagen GmbH, Hilden, Germany). 500 ng total RNA was changed to cDNA by miScript II RT kit (Qiagen, Inc., Valencia, CA, USA). QuantiTect SYBR Green PCR Master (Qiagen, Inc.) was used to Qpcr using a 7900HT thermocycler (Applied Biosystems; Thermo Fisher Scientific, Inc., Waltham, MA, USA). Qpcr were as follows: $50^{\circ} \mathrm{C}$ for $2 \mathrm{~min}, 95^{\circ} \mathrm{C}$ for $10 \mathrm{~min}$, followed by a third step for denaturation at $95^{\circ} \mathrm{C}$ for $15 \mathrm{sec}$ and at $60^{\circ} \mathrm{C}$ for 1 min repeated for 40 cycles. Data were presented as fold change in expression and were calculated as $2^{-\Delta \Delta \mathrm{Cq}}$.

Morris water maze teat. Cognitive function was evaluated using Morris water maze test after treatment with SRT1720. Rat was trained twice per day and the test was performed blindly for five days. Swimming study was video tracked, and latency, path length and swim speed. Then, cumulative distance at the platform was recorded. The mean time spent in the correct quadrant containing the platform after a probe trial and the mean number of time that mice crossed the former platform position during $1.5 \mathrm{~min}$ were analzyed.

In vitro model and transfection. PC12 cells were cultured in DMEM (HyClone; GE Healthcare Life Sciences, Logan, UT, USA) containing 10\% FBS (Gibco; Thermo Fisher Scientific, Inc.) at $37^{\circ} \mathrm{C}$ in $5 \% \mathrm{CO}_{2} .50 \mathrm{nM}$ of miRNA-23b-3p mimics (CTC AGGTGCTCTGGCTGCTTGGGTTCCTGGCATGCTGAT TTGTGACTTAAGATTAAAATCACATTGCCAGGGATT ACCACGCAACCACGACCTTGGC) and negative mimics (CCCCCCCCCCCCCC) were transfected into cell using with RNAiMAX (Thermo Fisher Scientific, Inc.). After transfection for $12 \mathrm{~h}, \mathrm{PC} 12$ cells $\left(1 \times 10^{6}\right.$ cell/per well) were treated with $25 \mathrm{mg} / \mathrm{ml}$ of glucose for 24,48 or $72 \mathrm{~h}$.

Cell Proliferation assay. After treatment with glucose for 24,48 or 72 h, PC12 cells $\left(1 \times 10^{3}\right.$ cell/per well) proliferation was measured using MTT assay (American Type Culture Collection, Manassas, VA, USA) for $4 \mathrm{~h}$ at $37^{\circ} \mathrm{C}$. DMSO assay was added into cell for $20 \mathrm{~min}$ at $37^{\circ} \mathrm{C}$. Absorbance was measured using ELISA reader (a Multiskan EX; Thermo Labsystems, Helsinki, Finland) at $490 \mathrm{~nm}$.

Apoptosis assay and Caspase-3/9 activity. After treatment with glucose for $48 \mathrm{~h}$, PC12 cells $\left(1 \times 10^{6}\right.$ cell/per well) was harvested and stained with $5 \mu \mathrm{l}$ Annexin V-PE and $5 \mu \mathrm{l}$ propidium iodide using an Apoptosis kit (BD Pharmingen, Franklin Lakes, NJ, USA) for 15 min at darkness. Apoptosis rate was measured using with a flow cytometer (FACSCalibur ${ }^{\mathrm{TM}}$ system; BD Biosciences, San Jose, CA, USA).

Caspase-3/9 activity was measured using Caspase-3/9 activity kits (Beyotime, Shanghai, China). Absorbance was measured using ELISA reader (a Multiskan EX; Thermo Labsystems) at $405 \mathrm{~nm}$.

Measurement of oxidatie stress. After treatment with glucose for $48 \mathrm{~h}$ or treatment with SIRT1 for 4 weeks, PC12 cells $\left(1 \times 10^{6}\right.$ cell/per well) or hippocampus tissue were collected and protein was splitted using RIPA assay and protein concentration was determined using BCA kit (Bio-Rad Laboratories, Inc., Hercules, CA, USA). $5 \mu \mathrm{g}$ protein was used to measure MDA, GSH, GSH-PX, and SOD levels using ELISA kits and absorbance was measured using ELISA reader (a Multiskan EX; Thermo Labsystems) at $450 \mathrm{~nm}$.

Western blot analysis. The hippocampus after treatment or PC12 cells ( $1 \times 10^{6}$ cell/per well) after transfection was splitted using RIPA assay and protein concentration was determined using BCA kit (Bio-Rad Laboratories, Inc.). Protein samples 


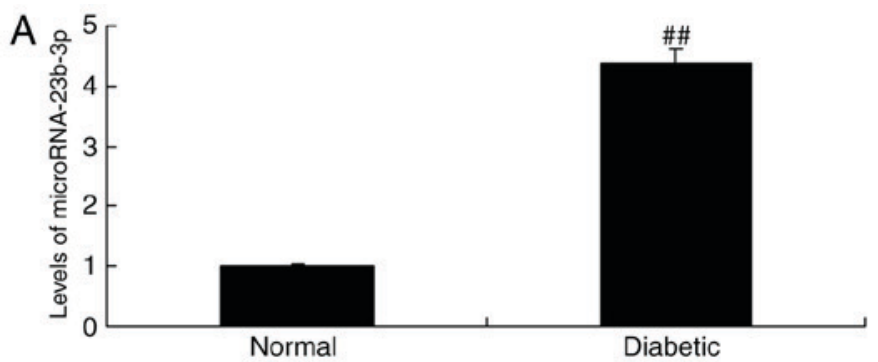

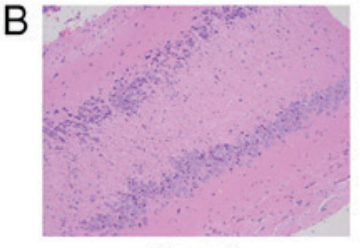

Normal

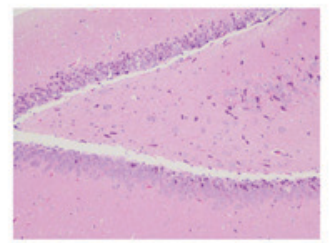

Diabetic

Figure 1. miRNA-23b-3p expression in diabetic rats. (A) miRNA-23b-3p expression and (B) H\&E staining (magnification, $\mathrm{x} 10$ ). ${ }^{\# \#} \mathrm{P}<0.01$ vs. normal (control) group. Normal, control rat group; Diabetic, diabetic rat group; miRNA, microRNA.
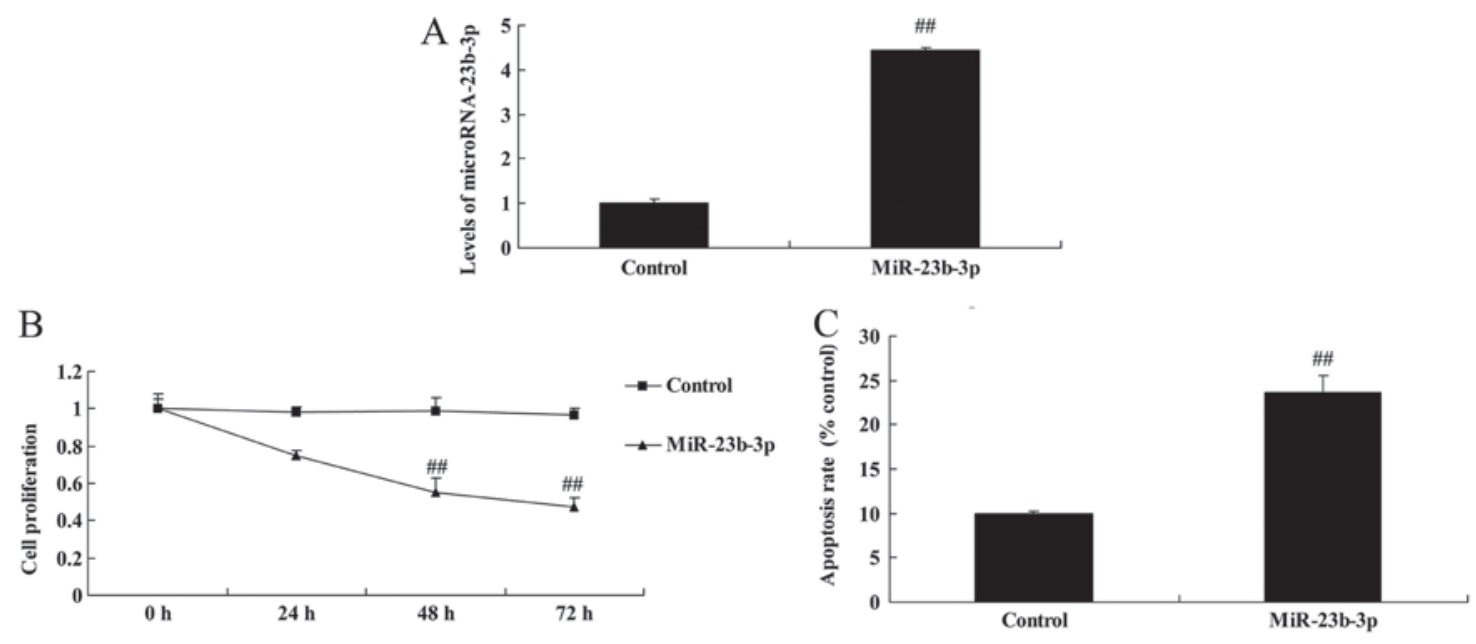

Figure 2. Overexpression of miRNA-23b-3p increased apoptosis in neurocytes following high glucose treatment. (A) miRNA-23b-3p expression, and levels of (B) cell proliferation and (C) apoptosis in neurocytes following high-glucose treatment. ${ }^{\# \#} \mathrm{P}<0.01$ vs. control group. Control, negative control group; miR-23b-3p, overexpression of microRNA-23b-3p group.

$(50 \mu \mathrm{g})$ were separated via $8-12 \%(\mathrm{w} / \mathrm{v})$ SDS-PAGE and transferred onto PVDF membranes. The membranes were blocked in PBST buffer containing 5\% (w/v) skimmed milk for $1 \mathrm{~h}$ at $37^{\circ} \mathrm{C}$ and incubated with Bax (sc-493, 1:500; Santa Cruz Biotechnology, Inc., Dallas, TX, USA), Sirt1 (sc-15404, 1:500; Santa Cruz Biotechnology, Inc.), nuclear factor erythroid 2-related factor 2 (Nrf2; sc-722, 1:500; Santa Cruz Biotechnology, Inc.) and GAPDH (sc-25778, 1:500; Santa Cruz Biotechnology, Inc.) overnight at $4^{\circ} \mathrm{C}$. The membranes were then incubated with the corresponding horseradish peroxidase-conjugated secondary antibody (7074, 1:5,000; Cell Signaling Technology, Inc., Danvers, MA, USA) at $37^{\circ} \mathrm{C}$ for $1 \mathrm{~h}$. The protein levels were measured using ECL kit (Bio-Rad Laboratories, Inc.) and quantified using Quantity One software version 4.62 (Bio-Rad Laboratories, Inc.).

Statistical analysis. All values are expressed as the mean \pm standard deviation. One-way analysis of variance followed by Dunnett's t-test was used for comparison between the groups. A two-tailed value of $\mathrm{P}<0.05$ was considered to indicate a statistically significant value.

\section{Results}

miRNA-23b-3p expression. In order to analyze the change levels of miRNA-23b-3p in cognitive impairment of diabetic rats, it may be a importance role for cognitive impairment of diabetic rats or neurocyte cell apoptosis. As shown in Fig. 1, miRNA-23b-3p expression was up-regulated, and neurocyte appeared dearth in cognitive impairment of diabetic rats, compared with normal control group.

Overexpression of miRNA-23b-3p increased apoptosis in neurocyte cell by high-glucose. We studied the possible implication of miRNA-23b-3p in neurocyte cell apoptosis by high-glucose. This result suggests miRNA-23b-3p mimics increased miRNA-23b-3p expression in neurocyte cell by high-glucose, compared with control group (Fig. 2A). Then, overexpression of miRNA-23b-3p inhibited cell proliferation, and increased apoptosis, Bax protein expression and caspase-3 activity in neurocyte cell by high-glucose, compared with control group (Figs. 2B, C and 3).

Overexpression of miRNA-23b-3p increased oxidative stress in neurocyte cell by high-glucose. Meanwhile, overexpression of miRNA-23b-3p also increased oxidative stress in neurocyte cell by high-glucose, compared with control group, which miRNA-23b-3p may mediated oxidative stress to induce neurocyte cell apoptosis (Fig. 4).

Overexpression of miRNA-23b-3p suppressed SIRT1 and Nrf2 protein expression in neurocyte cell by high-glucose. 

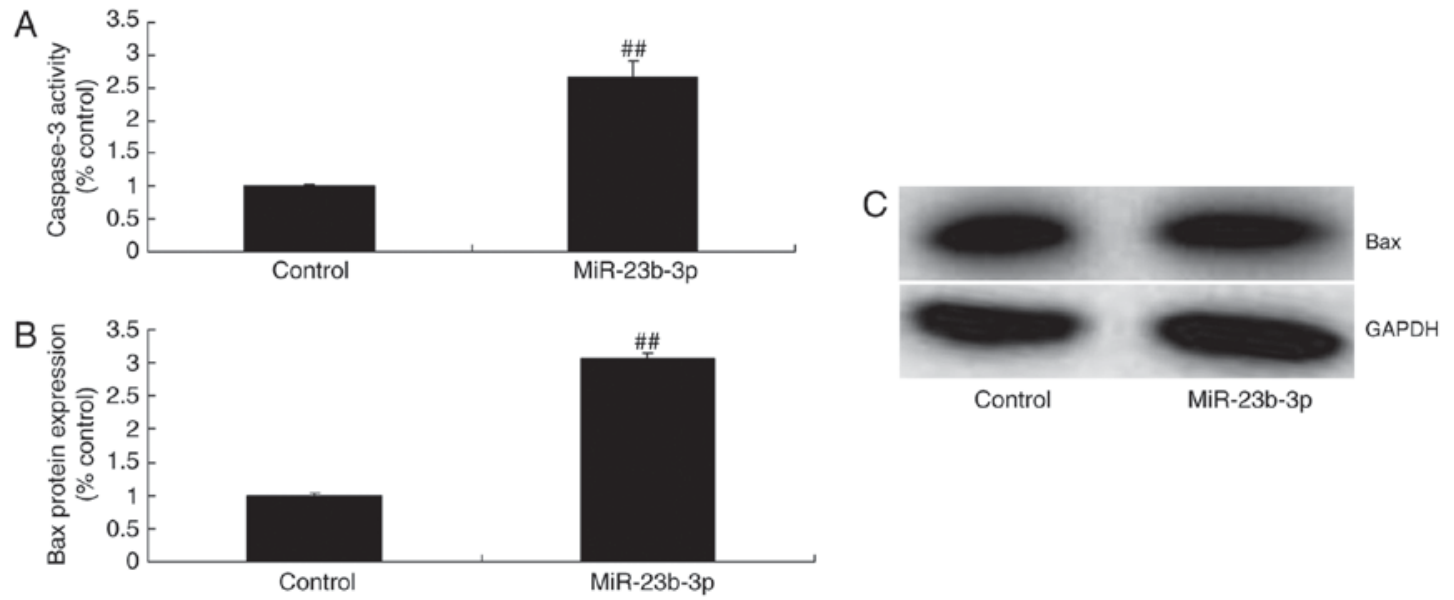

Figure 3. Overexpression of miRNA-23b-3p increased Bax protein expression and caspase-3 activity in neurocytes following high-glucose treatment. (A) Caspase-3 activity, and (B) Bax protein expression determined by (C) western blot analysis. ${ }^{\# \#} \mathrm{P}<0.01$ vs. control group. Control, negative control group; miR-23b-3p, overexpression of microRNA-23b-3p group; Bax, B-cell lymphoma 2-associated X protein.

A

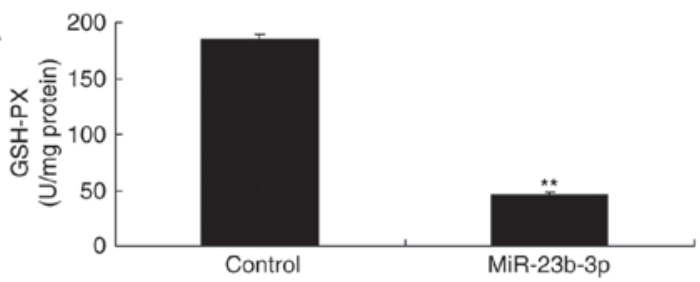

$\mathrm{C}$

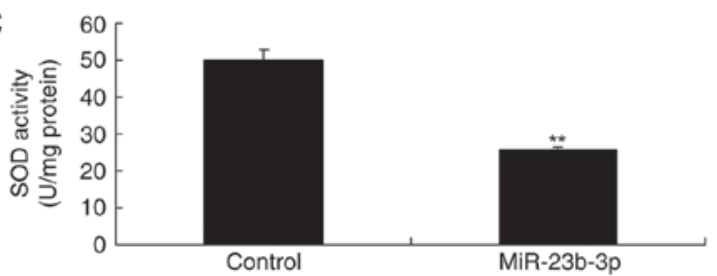

B

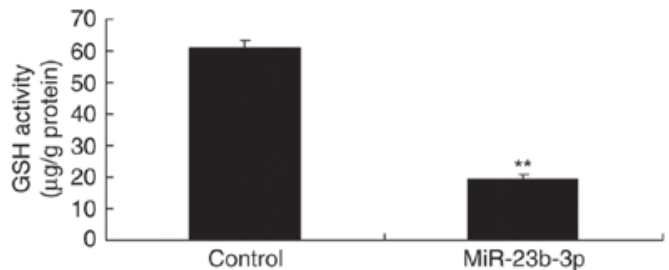

D

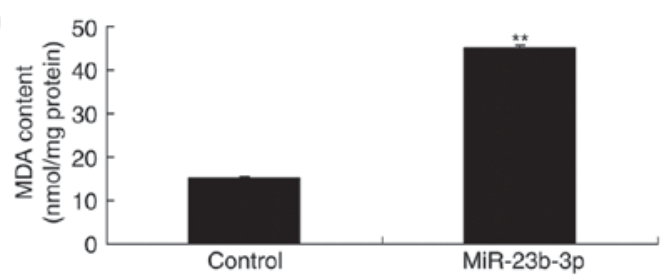

Figure 4. Overexpression of miRNA-23b-3p increased oxidative stress in neurocytes following high-glucose treatment. Levels of (A) GSH-PX, (B) GSH activity, (C) SOD activity and (D) MDA content. ${ }^{* *} \mathrm{P}<0.01$ vs. control group. Control, negative control group; miR-23b-3p, overexpression of microRNA-23b-3p group; GSH-PX, glutathione peroxidase; GSH, glutathione; SOD, superoxide dismutase; MDA, malondialdehyde.

We detected SIRT1 and Nrf2 protein expression to explore the mechanism of miRNA-23b-3p on oxidative stress, SIRT1 and Nrf2 protein expression were measured. Overexpression of miRNA-23b-3p suppressed SIRT1 and Nrf2 protein expression in neurocyte cell by high-glucose (Fig. 5). These results support the theory that miRNA-23b-3p an oncogene that may contribute to oxidative stress in neurocyte cell by high-glucose.

In vitro model of neurocyte cell by high-glucose, the promotion of SIRT1 induced SIRT1 and Nrf2 protein expression. SRT1720, SIRT1 agonist, $10 \mu \mathrm{M}$ for $48 \mathrm{~h}$, was added into cell following miRNA-23b-3p, and SIRT1 and Nrf2 protein expression were measured. Fig. 6 showed that SIRT1 agonist induced SIRT1 and Nrf2 protein expression in neurocyte cell by high-glucose following miRNA-23b-3p, compared with miRNA-23b-3p group.

In vitro model of neurocyte cell by high-glucose, the promotion of SIRT1 inhibited apoptosis. Then, in vitro model of neurocyte cell by high-glucose, the promotion of SIRT1 inhibited the suppression of cell proliferation, and activation of apoptosis rate, Bax and caspase-3 activity in neurocyte cell by high-glucose following miRNA-23b-3p, compared with miRNA-23b-3p group (Figs. 7 and 8).

In vitro model of neurocyte cell by high-glucose, the promotion of SIRT1 inhibited oxidative stress. Additionally, the promotion of SIRT1 inhibited the activation of MDA level, and inactivation of GSH, GSH-PX, and SOD levels in neurocyte cell by high-glucose following miRNA-23b-3p, compared with miRNA-23b-3p group (Fig. 9). In vitro model of neurocyte cell by high-glucose, the promotion of SIRT1 inhibited oxidative stress, which may be regulated neurocyte cell apoptosis to generate cognitive impairment of diabetic rats.

In vivo of diabetic rats, the promotion of SIRT1 induced SIRTI and Nrf 2 protein expression. So, we used vivo of diabetic rats to determine the function of SIRT1 on cognitive impairment of diabetic rats. SRT1720, SIRT1 agonist, $50 \mathrm{mg} / \mathrm{kg}, 4$ weeks, induced SIRT1 and Nrf2 protein expression in diabetic rats, compared with DM rat (Fig. 10). 


\section{A}

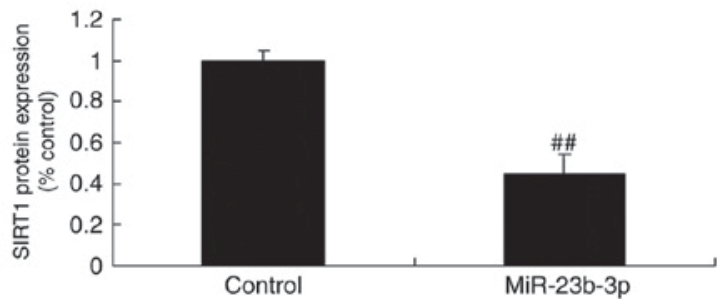

B

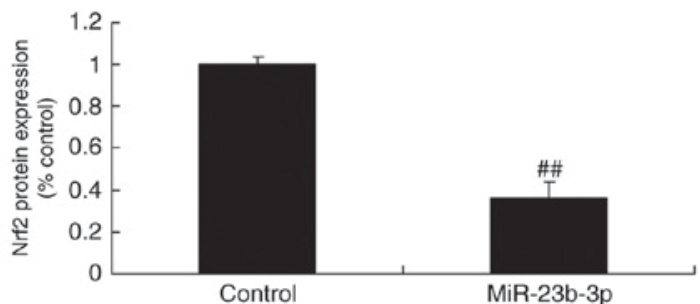

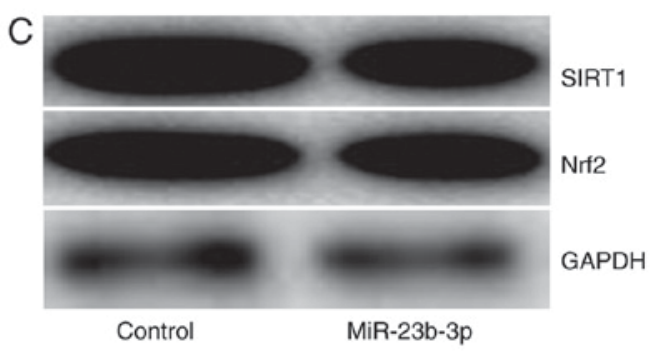

MiR-23b-3p

Figure 5. Overexpression of miRNA-23b-3p suppressed SIRT1 and Nrf2 protein expression in neurocytes following high-glucose treatment. (A) SIRT1 and (B) Nrf2 protein expression were determined by (C) western blot analysis. ${ }^{\# \#} \mathrm{P}<0.01$ vs. control group. Control, negative control group; miR-23b-3p, overexpression of microRNA-23b-3p group; SIRT1, silent information regulator 1; Nrf2, nuclear factor erythroid 2-related factor 2.

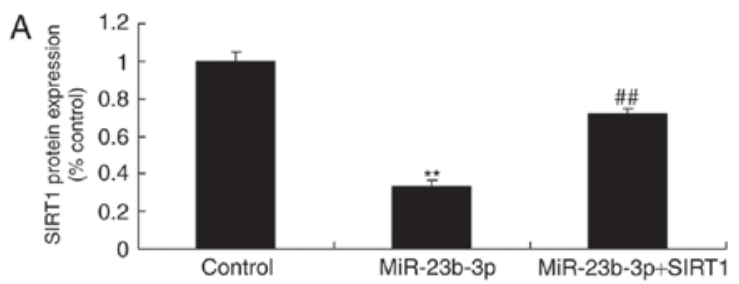

B

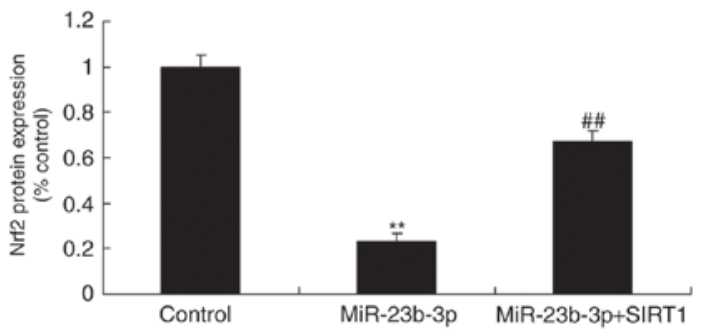

C

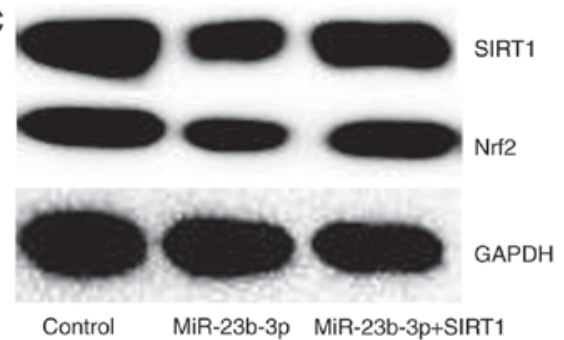

Control MiR-23b-3p MiR-23b-3p+SIRT1

Figure 6. An in vitro model of neurocytes treated with high levels of glucose and the promotion of SIRT1-induced SIRT1 and Nrf2 protein expression. (A) SIRT1 and (B) Nrf2 protein expression were determined by (C) western blot analysis. ${ }^{* *} \mathrm{P}<0.01$ vs. control group; ${ }^{\# \#} \mathrm{P}<0.01$ vs. miR-23b-3p group. Control, negative control group; miR-23b-3p, overexpression of microRNA-23b-3p group; miR-23b-3p+SIRT1, miRNA-23b-3p overexpression and SRT1720 (SIRT1 agonist) group; SIRT1, silent information regulator 1; Nrf2, nuclear factor erythroid 2-related factor 2.
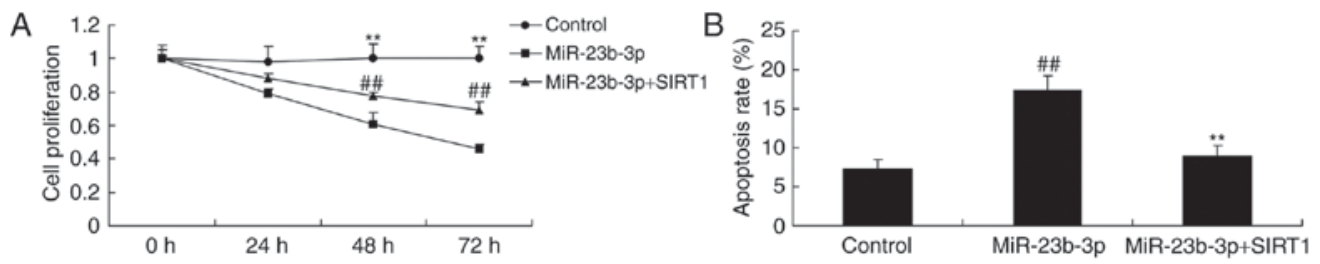

Figure 7. An in vitro model of neurocytes with high glucose treatment and the promotion of SIRT1-induced inhibition of apoptosis. Cell (A) proliferation and (B) apoptosis in neurocytes following high glucose treatment. ${ }^{\# \#} \mathrm{P}<0.01$ vs. control group; ${ }^{* *} \mathrm{P}<0.01 \mathrm{vs}$. miR-23b-3p group. Control, negative control group; miR-23b-3p, overexpression of microRNA-23b-3p group; miR-23b-3p+SIRT1, miRNA-23b-3p overexpression and SRT1720 (SIRT1 agonist) group; SIRT1, silent information regulator 1 .

In vivo of diabetic rats, the promotion of SIRT1 inhibited apoptosis and oxidative stress. The promotion of SIRT1 inhibited the activation of apoptosis rate, Bax and caspase-3/9 activity, and the induction of MDA level, and inhibition of GSH, GSH-PX, and SOD levels in diabetic rats, compared with DM rat (Figs. 11 and 12).
In vivo of diabetic rats, the promotion of SIRT1 prevents cognitive impairment. In order to assess the effect of SIRT1 on cognitive impairment of diabetic rats, Morris water maze teat was used to measure. As showed in Fig. 13A and B, the promotion of SIRT1 reduced shorter escape latency and the mean path length in diabetic rats, compared with DM rat. 

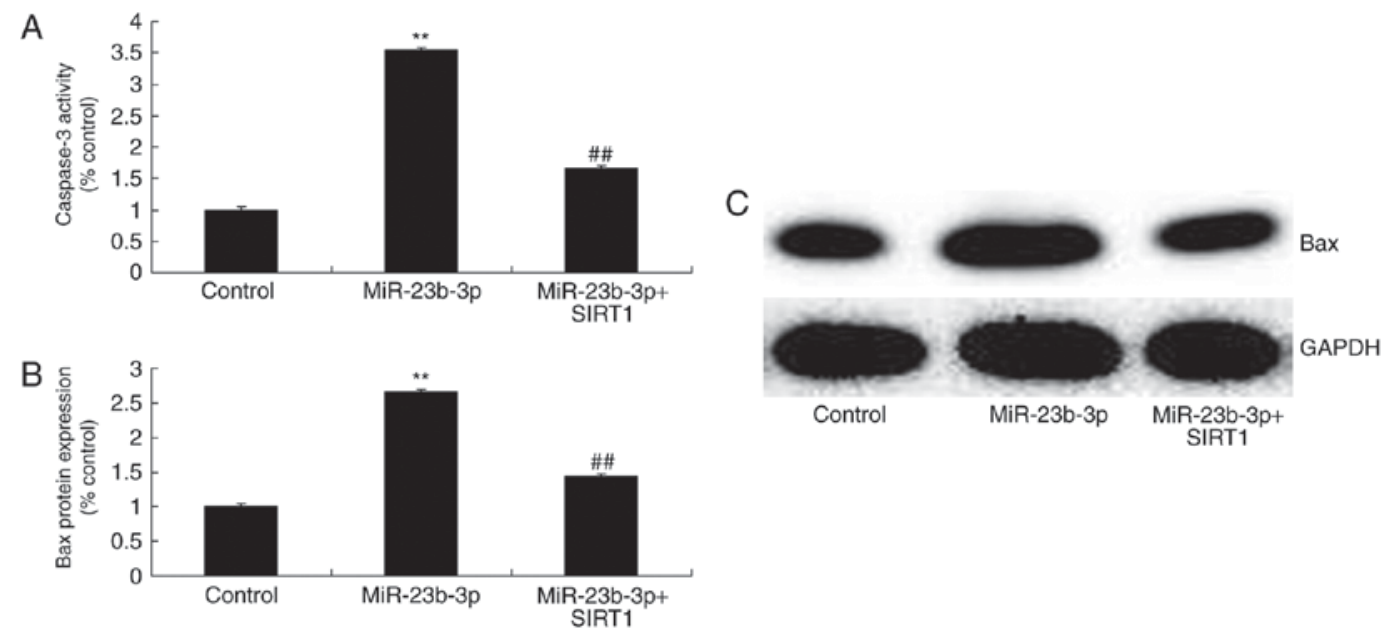

Figure 8. An in vitro model of neurocytes with high glucose treatment and the promotion of SIRT1-induced inhibition of Bax protein expression and caspase-3 activity. (A) Caspase-3 activity, and (B) Bax protein expression determined by (C) western blot analysis. ${ }^{* *} \mathrm{P}<0.01$ vs. control group; ${ }^{\# /} \mathrm{P}<0.01 \mathrm{vs}$. miR-23b-3p group. Control, negative control group; miR-23b-3p, overexpression of microRNA-23b-3p group; miR-23b-3p+SIRT1, miRNA-23b-3p overexpression and SRT1720 (SIRT1 agonist) group; SIRT1, silent information regulator 1; Bax, B-cell lymphoma 2-associated X protein.
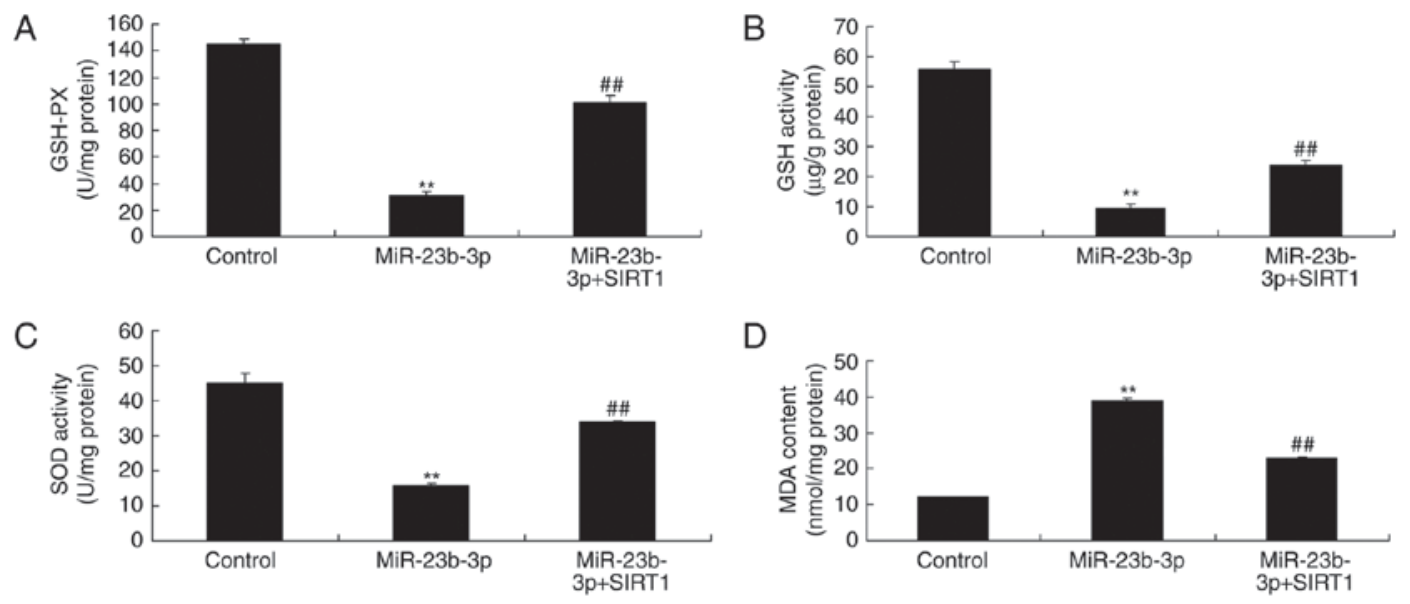

Figure 9. An in vitro model of neurocytes with high glucose treatment and the promotion of SIRT1-induced inhibition of oxidative stress. Levels of (A) GSH-PX, (B) GSH activity, (C) SOD activity and (D) MDA content. ${ }^{* *} \mathrm{P}<0.01$ vs. control group; ${ }^{\# /} \mathrm{P}<0.01$ vs. miR-23b-3p group. Control, negative control group; miR-23b-3p, overexpression of microRNA-23b-3p group; miR-23b-3p+SIRT1, miRNA-23b-3p overexpression and SRT1720 (SIRT1 agonist) group; SIRT1, silent information regulator 1; GSH-PX, glutathione peroxidase; GSH, glutathione; SOD, superoxide dismutase; MDA, malondialdehyde.
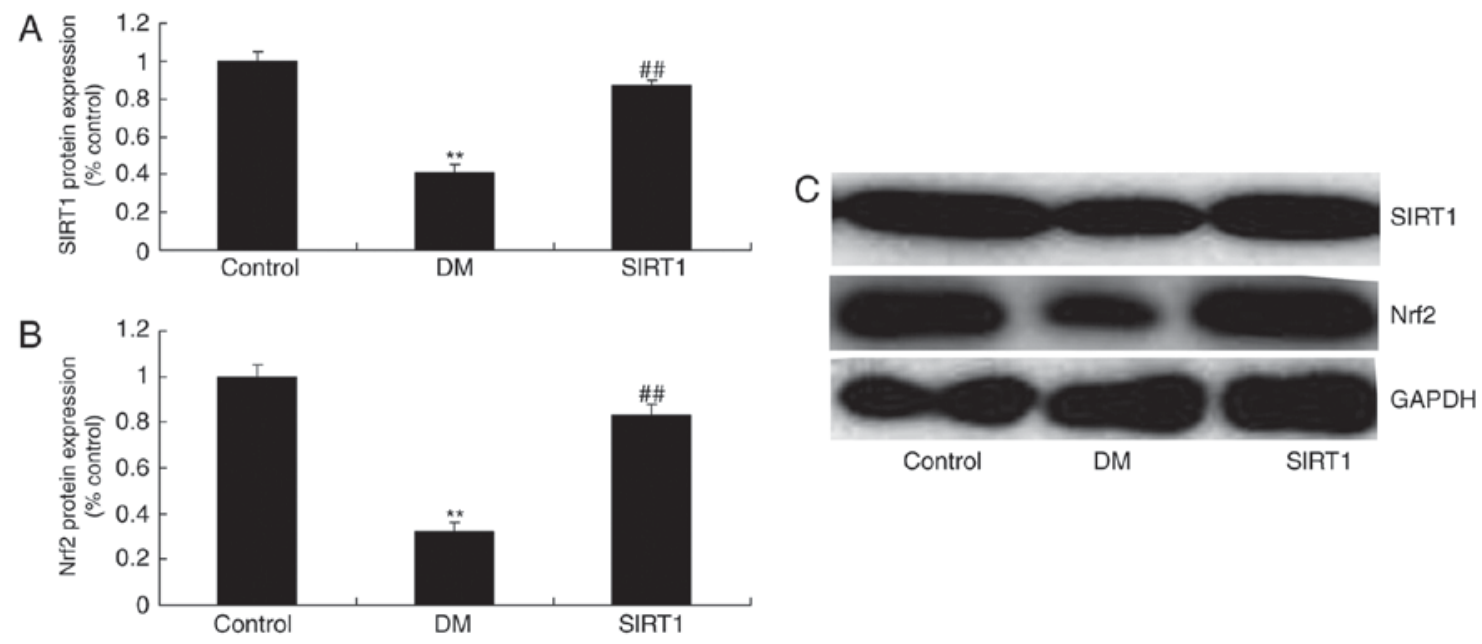

Figure 10. An in vivo model of diabetes in rats and the promotion of SIRT1-induced SIRT1 and Nrf2 protein expression. (A) SIRT1 and (B) Nrf2 protein expression were determined by $(\mathrm{C})$ western blot analysis. ${ }^{* *} \mathrm{P}<0.01$ vs. control group; ${ }^{\# \#} \mathrm{P}<0.01 \mathrm{vs.} \mathrm{DM} \mathrm{group.} \mathrm{Control,} \mathrm{negative} \mathrm{control} \mathrm{group;} \mathrm{DM,} \mathrm{diabetic} \mathrm{rat}$ group; SIRT1, silent information regulator 1, agonist SRT1720 treatment group; Nrf2, nuclear factor erythroid 2-related factor 2. 
A
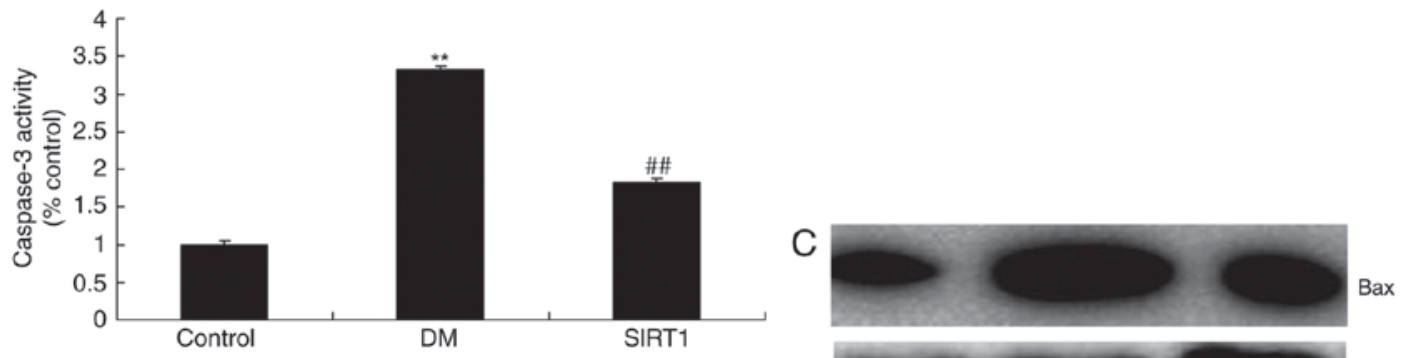

B
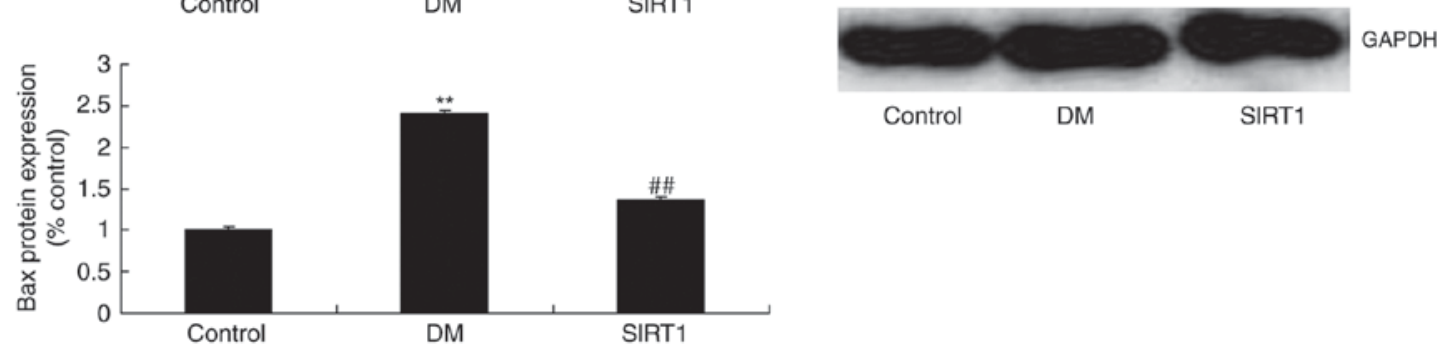
expression determined by (C) western blot analysis. ${ }^{* *} \mathrm{P}<0.01$ vs. control group; ${ }^{\# \prime} \mathrm{P}<0.01$ vs. DM group. Control, negative control group; DM, diabetic rat group; SIRT1, silent information regulator 1, agonist SRT1720 treatment group; Bax, B-cell lymphoma 2-associated X protein.

A

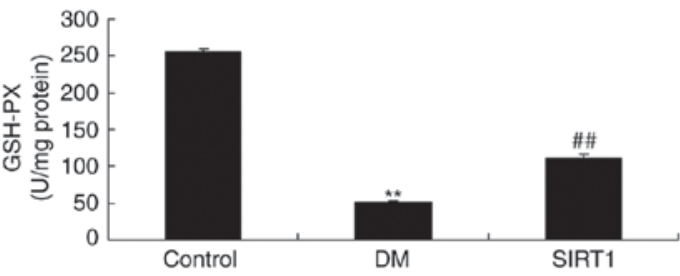

C

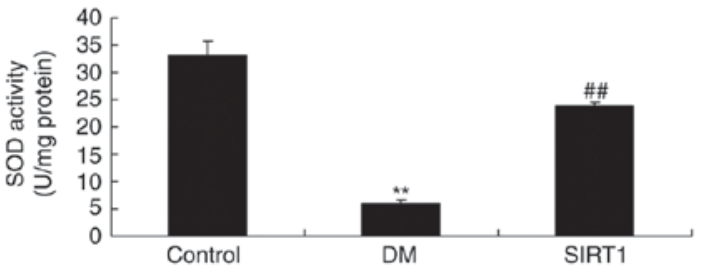

$\mathrm{B}$

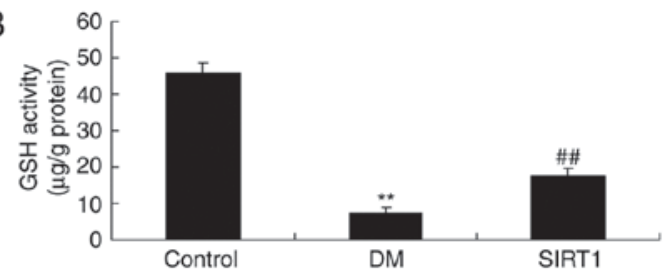

D

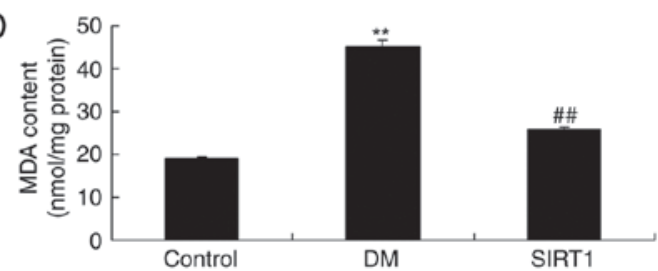

Figure 12. An in vivo model of diabetes in rats and the promotion of SIRT1-induced inhibition of oxidative stress. Levels of (A) GSH-PX, (B) GSH activity, (C) SOD activity and (D) MDA content. ${ }^{* *} \mathrm{P}<0.01$ vs. control group; ${ }^{\# \#} \mathrm{P}<0.01$ vs. DM group. Control, negative control group; DM, diabetic rat group; SIRT1, silent information regulator 1, agonist SRT1720 treatment group; GSH-PX, glutathione peroxidase; GSH, glutathione; SOD, superoxide dismutase; MDA, malondialdehyde.

A
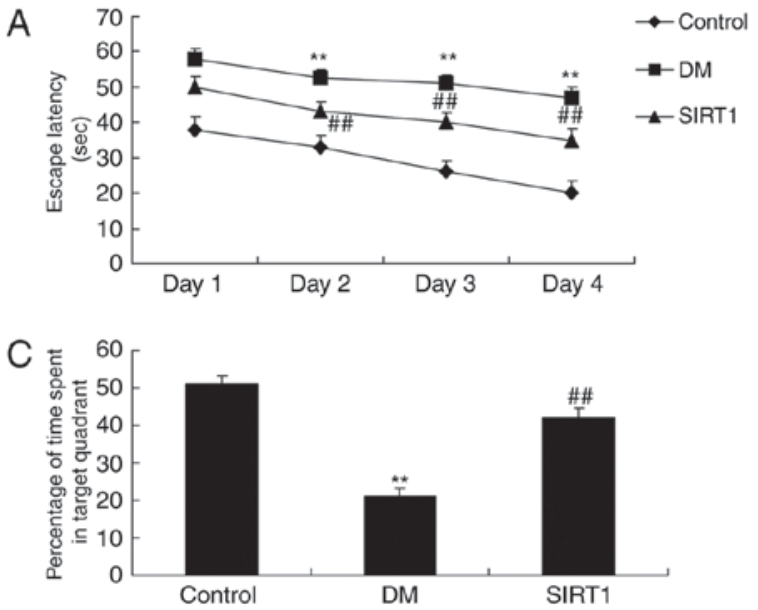

B

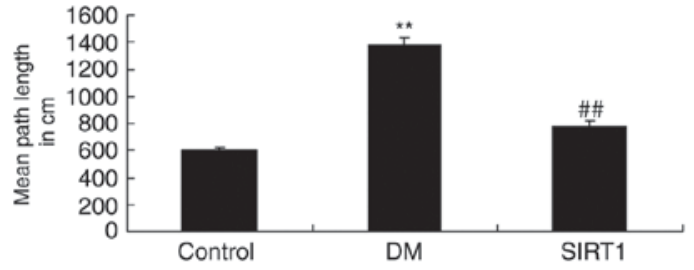

D

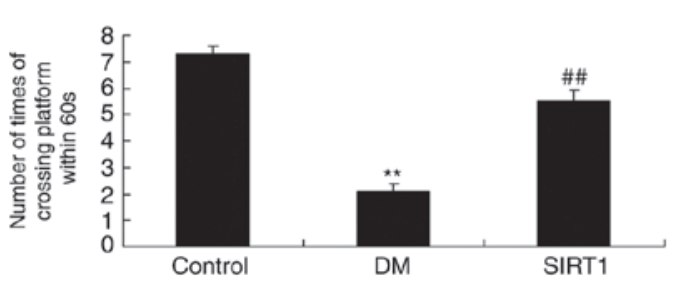

Figure 13. An in vivo model of diabetes in rats and the promotion of SIRT1 associated prevention of cognitive impairment. (A) The escape latency, (B) mean path length, $(\mathrm{C})$ the percentage of time spent in the target quadrant and (D) the number of times the animals crossed the former platform location. ${ }^{* *} \mathrm{P}<0.01$ vs. control group; ${ }^{\# \#} \mathrm{P}<0.01$ vs. DM group. Control, negative control group; DM, diabetic rat group; SIRT1, silent information regulator 1, agonist SRT1720 treatment group. 
Meanwhile, DM rats spent less time in the target quadrant and the number of times the animals crossed the former platform location were increased by the promotion of SIRT1, compared with DM rat (Fig. 13C and D).

\section{Discussion}

DM is a chronic and lifelong disease. Patients should master DM monitoring knowledge and detect all indexes regularly (15). This has played a vital role in reducing the incidence of complications in DM patients, as well as DM disability and fatality rates (15). DM cognitive impairment is a complication characterized by DM-induced cognitive impairment, accompanying with intracerebral structural and pathophysiological changes (16). The study suggested that miRNA-23b-3p expression was up-regulated, and neurocyte appeared dearth in cognitive impairment of diabetic rats. In this study, we only used Morris water maze to analyze cognitive impairment, which is a limitation of the current study, and we will execute more tests to measure the changes of cognitive impairment in further study.

Some scholars have suggested renaming it as DM encephalopathy and DM-related cognitive decline (17). But no consensus has been reached so far. Its pathogenesis and pathophysiological changes remain unclear. Previous research on glucose toxicity, insulin signal disorder, unbalanced homeostasis, inflammation, oxidative stress injury, vascular disease, and hypothalamus-pituitary-adrenal axis abnormality, has attained certain progress (17). We found that overexpression of miRNA-23b-3p inhibited cell proliferation, and increased apoptosis, Bax protein expression and caspase-3 activity in neurocyte cell by high-glucose. Zhan et al indicate that that miR-23b-3p supplement may be a potential anabolic in kainic acid-induced seizure (18).

Oxidative stress refers to dysfunction of antioxidant defense system, which leads to excessive production of reactive oxygen species and reactive nitrogen species, thus leading to tissue and cell injury (17). Oxidative stress is indicated in research to participate in DM genesis and development. It can also induce reduced nerve conduction velocity and promote neuronal apoptosis in DM mice (4). This suggests that oxidative stress is involved in DM-induced neuronal injury (4). In the present study, overexpression of miRNA-23b-3p also increased oxidative stress in neurocyte cell by high-glucose. Jiang et al reported that microRNA-23b-3p up-regulate reactive oxygen species (ROS) and oxidative stress in acute myeloid leukemia (19).

Oxidative stress is a pathological state caused by excessive production of oxygen free radical (17). This has given rise to excessive accumulation of oxygen free radical and related metabolites, thus producing all kinds of cytotoxicities (20). The occurrence of oxidative stress can also induce cell aging. SIRT1 has been verified to exert antioxidation through regulating the transcription activity of some key enzymes (21).

SIRT1 can protect pancreas islet $\beta$ cell, regulate insulin release, improve insulin resistance, reduce inflammatory response and regulate lipid metabolism (22). Thus, it can participate in regulating glucose homeostasis and thereby inhibit DM and DM-induced organ dysfunction (7). SIRT1 is indicated in research to alleviate DM genesis and DM-induced complications (23). Therefore, SIRT1 may become a target for treating insulin resistance and type 2 DM (23). The results showed that Overexpression of miRNA-23b-3p suppressed SIRT1 and Nrf2 protein expression in neurocyte cell by high-glucose. Zhao et al identified that miR-23b-3p induces the cellular metabolic memory of high glucose via SIRT1 pathway in diabetic retinopathy (24).

$\mathrm{Nrf} 2$ is the most important transcription factor for cell to regulate antioxidant stress response (24). Nrf2 activation is the precondition to exert its function (25). In contrast, Nrf2 dissociation with Keapl is the first step for it to regulate target gene transcription (26). Under physiological status, binding of Nrf2 with cytoplasmic partner Keap1 is relatively inhibited. Two cysteine sites on Keapl, C273 and C288, are simultaneously modified under the action of oxidative stressor. This has resulted in coupling of Keapl with Nrf2 (26). Meanwhile, the ubiquitination of ubiquitinating enzyme on $\mathrm{Nrf2}$ is weakened or eliminated (27). This leads to uncoupling of Nrf2 with Keapl, followed by nuclear translocation. Nrf2 then binds with the antioxidant response element ARE (27). Subsequently, it initiates the downstream antioxidant gene and expression of phase-II detoxifying enzyme (27). Thus, it enhances cell resistance to oxidative damage (28). Downstream antioxidant enzyme expression is reduced in the presence of disordered or deleted Nrf2 activation (29). Thus, it cannot act against toxicity of antioxidant stressor on cell. Consequently, it will lead to cell dysfunction, apoptosis or necrosis (29). We also showed that the promotion of SIRT1 inhibited apoptosis and oxidative stress, decreased the miRNA-23b-3p on cognitive impairment of diabetic rats via Nrf2 expression. Zhao et al showed that miR-23b-3p induces the cellular metabolic memory through a SIRT1-dependent signalling pathway in diabetic retinopathy (24). However, we only used Western blot to analyze the protein expression of SIRT1 by miR-23b-3p, which is a limitation of the current study, and we will execute more tests to measure the changes of SIRT1 and Nrf2 in further study.

In conclusion, the above histopathological and molecular biological results suggested that the Sirt1/Nrf2 signalling pathway is involved in oxidative stress-induced apoptosis and caused cognitive impairment in of diabetic rats through miR-23b-3p. Enhancing Sirt1/Nrf2 signalling pathway activity or the inhibition of miR-23b-3p may serve as a potential therapeutic strategy for cognitive impairment in diabetic.

\section{References}

1. Liu J, Guo B, Chen Z, Wang N, Iacovino M, Cheng J, Roden C, Pan W, Khan S, Chen S, et al: miR-125b promotes MLL-AF9-driven murine acute myeloid leukemia involving a VEGFA-mediated non-cell-intrinsic mechanism. Blood 129: 1491-1502, 2017.

2. Buo AM, Tomlinson RE, Eidelman ER, Chason M and Stains JP: Connexin43 and Runx2 interact to affect cortical bone geometry, skeletal development and osteoblast and osteoclast function. J Bone Miner Res 32: 1727-1738, 2017.

3. Bu Q, You F, Pan G, Yuan Q, Cui T, Hao L and Zhang J: miR-125b inhibits anaplastic thyroid cancer cell migration and invasion by targeting PIK3CD. Biomed Pharmacother 88: 443-448, 2017.

4. Shrivats AR, Hsu E, Averick S, Klimak M, Watt AC, DeMaio M, Matyjaszewski K and Hollinger JO: Cationic Nanogel-mediated Runx 2 and Osterix siRNA delivery decreases mineralization in MC3T3 Cells. Clin Orthop Relat Res 473: 2139-2149, 2015.

5. Fang Y, Gao F, Hao J and Liu Z: microRNA-1246 mediates lipopolysaccharide-induced pulmonary endothelial cell apoptosis and acute lung injury by targeting angiotensin-converting enzyme 2. Am J Transl Res 9: 1287-1296, 2017. 
6. Chen S, Feng B, Thomas AA and Chakrabarti S: miR-146a regulates glucose induced upregulation of inflammatory cytokines extracellular matrix proteins in the retina and kidney in diabetes. PLoS One 12: e0173918, 2017.

7. Zheng J, Lin Z, Dong P, Lu Z, Gao S, Chen X, Wu C and Yu F: Activation of hepatic stellate cells is suppressed by microRNA-150. Int J Mol Med 32: 17-24, 2013.

8. Yu B, Lv X, Su L, Li J, Yu Y, Gu Q, Yan M, Zhu Z and Liu B: miR-148a functions as a tumor suppressor by targeting CCK-BR via inactivating STAT3 and Akt in human gastric cancer. PLoS One 11: e0158961, 2016.

9. Sato T, Liu X, Nelson A, Nakanishi M, Kanaji N, Wang X, Kim M, Li Y, Sun J, Michalski J, et al: Reduced miR-146a increases prostaglandin $\mathrm{E}_{2}$ in chronic obstructive pulmonary disease fibroblasts. Am J Respir Crit Care Med 182: 1020-1029, 2010.

10. Li D, Duan M, Feng Y, Geng L, Li X and Zhang W: miR-146a modulates macrophage polarization in systemic juvenile idiopathic arthritis by targeting INHBA. Mol Immunol 77: 205-212, 2016.

11. Li HP, Huang HY, Lai YR, Huang JX, Chang KP, Hsueh C and Chang YS: Silencing of miRNA-148a by hypermethylation activates the integrin-mediated signaling pathway in nasopharyngeal carcinoma. Oncotarget 5: 7610-7624, 2014

12. Ye EA and Steinle JJ: miR-146a attenuates inflammatory pathways mediated by TLR4/NF- $\kappa \mathrm{B}$ and TNF $\alpha$ to Protect primary human retinal microvascular endothelial cells grown in high glucose. Mediators Inflamm 2016: 3958453, 2016.

13. Wu X, Long L, Liu J, Zhang J, Wu T, Chen X, Zhou B and Lv TZ: Gambogic acid suppresses inflammation in rheumatoid arthritis rats via PI3K/Akt/mTOR signaling pathway. Mol Med Rep 16: 7112-7118, 2017.

14. Liu B, Li F, Shi J, Yang D, Deng Y and Gong Q: Gastrodin ameliorates subacute phase cerebral ischemia-reperfusion injury by inhibiting inflammation and apoptosis in rats. Mol Med Rep 14: 4144-4152, 2016.

15. Lee M, Arikawa K and Nagahama F: Micromolar levels of sodium fluoride promote osteoblast differentiation through Runx2 signaling. Biol Trace Elem Res 178: 283-291, 2017.

16. Park OJ, Kim J, Yang J, Yun CH and Han SH: Muramyl dipeptide, a shared structural motif of peptidoglycans, is a novel inducer of bone formation through induction of Runx2. J Bone Miner Res 32: 1455-1468, 2017.

17. Choi YH, Kim GS, Choi JH, SW, Kim HG, Han Y, Lee DY, Choi SI, Kim SY, Ahn YS, et al: Ethanol extract of Lithospermum erythrorhizon Sieb. et Zucc. promotes osteoblastogenesis through the regulation of Runx2 and Osterix. Int J Mol Med 38: 610-618, 2016.
18. Zhan L, Yao Y, Fu H, et al: Protective role of miR-23b-3p in kainic acid-induced seizure. Neuroreport 27: 764-768, 2016

19. Jiang W, Min J, Sui X, et al: MicroRNA-26a-5p and microRNA-23b-3p up-regulate peroxiredoxin III in acute myeloid leukemia. Leuk Lymphoma 56: 460-471, 2015.

20. Sun Z, Cao X, Hu Z, Zhang L, Wang H, Zhou H, Li D, Zhang S and Xie M: miR-103 inhibits osteoblast proliferation mainly through suppressing Cav1.2 expression in simulated microgravity. Bone 76: 121-128, 2015.

21. Ozeki N, Hase N, Hiyama T, Yamaguchi H, Kawai-Asano R, Nakata K and Mogi M: MicroRNA-211 and autophagy-related gene 14 signaling regulate osteoblast-like cell differentiation of human induced pluripotent stem cells. Exp Cell Res 352: 63-74, 2017.

22. Li P, Sun N, Zeng J, Zeng Y, Fan Y, Feng W and Li J: Differential expression of miR-672-5p and miR-146a-5p in osteoblasts in rats after steroid intervention. Gene 591: 69-73, 2016.

23. Hata A and Kang H: Functions of the bone morphogenetic protein signaling pathway through microRNAs (Review). Int J Mol Med 35: 563-568, 2015.

24. Zhao S, Li T, Li J, Lu Q, Han C, Wang N, Qiu Q, Cao H, Xu X, Chen $\mathrm{H}$ and Zheng Z: miR-23b-3p induces the cellular metabolic memory of high glucose in diabetic retinopathy through a SIRT1-dependent signalling pathway. Diabetologia 59: 644-654, 2016.

25. Pan W, Miao L, Lin Y, Huang X, Ge X, Moosa SL, Liu B, Ren M, Zhou Q, Liang H, et al: Regulation mechanism of oxidative stress induced by high glucose through PI3K/Akt/Nrf2 pathway in juvenile blunt snout bream (Megalobrama amblycephala). Fish Shellfish Immunol 70: 66-75, 2017.

26. Wang XR, Shi GX, Yang JW, Yan CQ, Lin LT, Du SQ, Zhu W, He T, Zeng XH, Xu Q and Liu CZ: Acupuncture ameliorates cognitive impairment and hippocampus neuronal loss in experimental vascular dementia through Nrf2-mediated antioxidant response. Free Radic Biol Med 89: 1077-1084, 2015.

27. Wang Z, Ji C, Wu L, Qiu J, Li Q, Shao Z and Chen G: Tert-butylhydroquinone alleviates early brain injury and cognitive dysfunction after experimental subarachnoid hemorrhage: role of Keap1/Nrf2/ARE pathway. PLoS One 9: e97685, 2014

28. Wan P, Su W, Zhang Y, Li Z, Deng C and Zhuo Y: Trimetazidine protects retinal ganglion cells from acute glaucoma via the Nrf2/Ho-1 pathway. Clin Sci (Lond) 131: 2363-2375, 2017.

29. Guo Y, Sun J, Li T, Zhang Q, Bu S, Wang Q and Lai D Melatonin ameliorates restraint stress-induced oxidative stress and apoptosis in testicular cells via NF- $\mathrm{kB} / \mathrm{iNOS}$ and Nrf2/ HO-1 signaling pathway. Sci Rep 7: 9599, 2017. 